RESEARCH ARTICLE

\title{
Association between Intervention Delivery Approach for Postna- tal Depression and its Subsequent Adherence
}

\author{
Omobolanle Omisade*, Alice Good and Tineke Fitch \\ School of Computing, University of Portsmouth, Portsmouth, United Kingdom

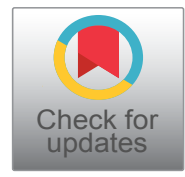

*Corresponding author: Omobolanle Omisade, School of Computing, University of Portsmouth, Portsmouth, United Kingdom, E-mail: omobolanle.omisade@port.ac.uk

\begin{abstract}
Background: Recent research on providing support for women with postnatal depression indicates that many women poorly comply with treatment or show only moderate improvement in depression. This paper reviews evidence on the association between intervention delivery approach for postnatal depression and its subsequent adherence.

Methods: In this review, we identified studies that address the relationship between intervention delivery, and adherence. Selected keywords were used as well as a manual search through the references of appropriate literature, yielding a total of 25 papers for this review.

Results: One suggested explanation for non-adherence to postnatal depression interventions is the delivery approach. This review indicates that the availability of a flexible delivery option may encourage long-term treatment compliance. Due to depressed women's overwhelming responsibilities, an adequate provision for infant care during the treatment process can increase adherence. We also identified that engaging women during treatment session might facilitate high compliance to treatment.

Limitation: Measures of adherence and time points varied considerably in included studies, and the selected keywords may have led to the omission of relevant references.

Conclusion: Non-adherence to postnatal depression intervention should not be seen as the patient's problem as it may represent a fundamental limitation in the delivery of postnatal depression interventions. We advise further research in providing adjunct support to postnatal depression treatments. Adjunct treatment support could give users access to additional wellbeing support and help overcome obstacles to treatment compliance.
\end{abstract}

\section{Keywords}

Postnatal depression, Treatment delivery, Treatment adherence

\begin{abstract}
Abbreviations
BDI: Beck's Depression Inventory; CSS: Cross-Sectional Survey; EPDS: Edinburgh Postpartum Depression Scale; HRS-D: Hamilton Rating Scale for Depression; HV: Health Visitor; MADRS: Montgomery-Asberg Depression Rating Scale; MCP: Multicomponent intervention; NR: Not Reported; PH: Participant's Home; PT: Participant Telephone/ Mobile Phone; PCA: Person Centered Approach; PMI: Pharmacological Intervention; PCC: Primary Care Centre; PCN: Primary Care/Early Childhood Nurse; PHI: Psychological Intervention; RCT: Randomised Control Trial; RC: Researcher; SH: Self-Help; SSI: Semi Structured Interview; HP: Therapist, Psychiatrist, GP, Clinicians; UC: Usual Care; FF: Face to Face
\end{abstract}

\section{Introduction}

Postnatal depression (PND) is defined in this study as a non-psychotic depressive episode which meets standardised diagnostic criteria for a minor or major depressive disorder, beginning in or extending into the postnatal period, and is usually defined at up to 12 months postpartum [1-6]. There is evidence that PND affects $10-15 \%$ of mothers and can lead to cognitive, and emotional disturbance for the baby in addition to the adverse effects on the mother [7,8]. Due to the negative consequences of PND on mothers, infants, and their family, effective treatments are needed to prevent these problems [7].

Studies have suggested effective pharmacological and psychological interventions for the treatment of PND [7,9-15]. For example, one study compared antidepressants and a placebo intervention for the treatment of PND establishing an improvement in overall

Citation: Omisade O, Good A, Fitch T (2018) Association between Intervention Delivery Approach for Postnatal Depression and its Subsequent Adherence. Int J Womens Health Wellness 4:065. doi. org/10.23937/2474-1353/1510065

Received: May 09, 2017: Accepted: January 10, 2018: Published: January 12, 2018

Copyright: (C) 2018 Omisade O, et al. This is an open-access article distributed under the terms of the Creative Commons Attribution License, which permits unrestricted use, distribution, and reproduction in any medium, provided the original author and source are credited. 
clinical severity found for the antidepressant group in comparison with the controlled group [14]. It has been suggested by another study that counselling informed by the principles of Cognitive Behavioural Therapy (CBT) is effective when delivered by maternal and child health nurses [16]. Furthermore, Interpersonal Therapy (IPT) has also been found to be effective for this population [17]. However, the postnatal period still presents specific barriers to adequate improvement in depression, and a large number of women remain without treatment, poorly adhere to the prescribed intervention or show only moderate improvement in depression [18-20].

Adherence is defined as the extent to which a person's behaviour conforms to medical or health advice $[21,22]$. A systematic review on patient adherence in the treatment of depression confirmed that adherence is a major problem with one in three patients not completing their prescribed treatment $[20,22]$. Receiving treatment for PND and sustaining it over the long-term has been proven to be very difficult due to barriers. These barriers could include struggles with variable infant feeding demands and napping schedules that may interfere with regular appointment attendance [23]. Some women face difficulty in looking after their children due to impaired state of mind; maternal sleep difficulties that may be associated with infant sleep schedules and problems adjusting to and managing the infant needs balanced against other valued tasks [19,23-28]. Poor patient-doctor relationship, negative experiences with seeking help in the past, long waiting list for treatments, location convenience, and perceived stigma can also lead to women avoiding treatments and interfere with the ability of women to attend regular scheduled appointment [22,26,29-33]. For example, in a study on the efficacy of group Interpersonal Therapy carried out by Klier and colleagues, five of 22 eligible women chose not to participate and attributed unwillingness to transportation or childcare difficulties [17]. The delivery of psychological intervention may prove difficult especially when balancing the demands of caring for infants or other children with treatment process and efforts required in seeking help or treatment compliance [34-37]. The transportation problem and childcare difficulties highlighted in these studies are consistent with the findings of other authors $[25,36]$. These might be as a result of the delivery location or schedule [20]. However, the effect of delivery location of PND adherence needs to be further investigated and addressed [38].

Intervention delivery appears to be a possible contributor to non-adherence of PND treatments. Several studies have reported that delivery of an intervention can have an effect on its subsequent adherence [12,19,22-25,33,39-41]. In a qualitative study on women's experience with online self-help Computerised Cognitive Behavioural Therapy (CCBT) program, adherence to treatment was reported low, and this was attributed to the way of treatment delivery and lack to support to adhere with intervention [32]. Intervention delivery should be considered essential for sustaining treatment effect over the long-term [2,32,42]. Hence, providing a means for women with PND to easily obtain access to a high-quality intervention delivery, which encourages engagement and compliance, may be beneficial. The availability of additional support for effective PND interventions might help provide the support needed to adhere to treatment and encourage just-in-time intervention delivery, adherence and support for coping with the demands associated with childcare.

However, to the best of our knowledge existing studies have not specially examined the association between delivery of intervention and its subsequent adherence to PND. The focus of this paper is to review research on the association between the delivery and adherence to intervention used to support women with PND. This knowledge will help to decide on the factors that should be considered by an adjunct support that might have the potential to facilitate adherence to PND interventions. We envisage that adjunct support could give postnatal depressed women access to supplementary wellbeing support, motivate them to self-manage as well as overcome obstacles to treatment compliance.

\section{Methods}

Studies were identified by searching electronic databases, which included: PubMed; IEEE; British Medical Journal; British Journal of Psychiatry; Journal of Clinical Psychology; Journal of Affective Disorder and Medline. These databases were used because of their relevance to the topic of PND and PND support interventions. Throughout this review, the terms intervention, treatment, and therapy are used interchangeably to mean pharmacological, psychological or multicomponent (multiple treatments) treatments for women with PND. For this paper, the term compliance means adherence to treatment. Studies were included if they reported information related to intervention adherence; compliance; delivery and/or treatment preferences and if they focused on postnatal or postpartum depression. From the 1326 titles and abstract identified, duplicate references were removed, and 805 non-relevant references were excluded. A total of 25 articles were judged potentially eligible for this review and reading. The study seeks to answer the research questions below:

1. Is intervention delivery associated with adherence?

2. What are the factors that determine the association between intervention delivery and its subsequent adherence?

\section{Search methodology}

The search keywords used included a combination of "postnatal depression"; "postpartum depression"; "intervention"; "adherence with treatment", and "delivery of treatment". The search result was restricted to 


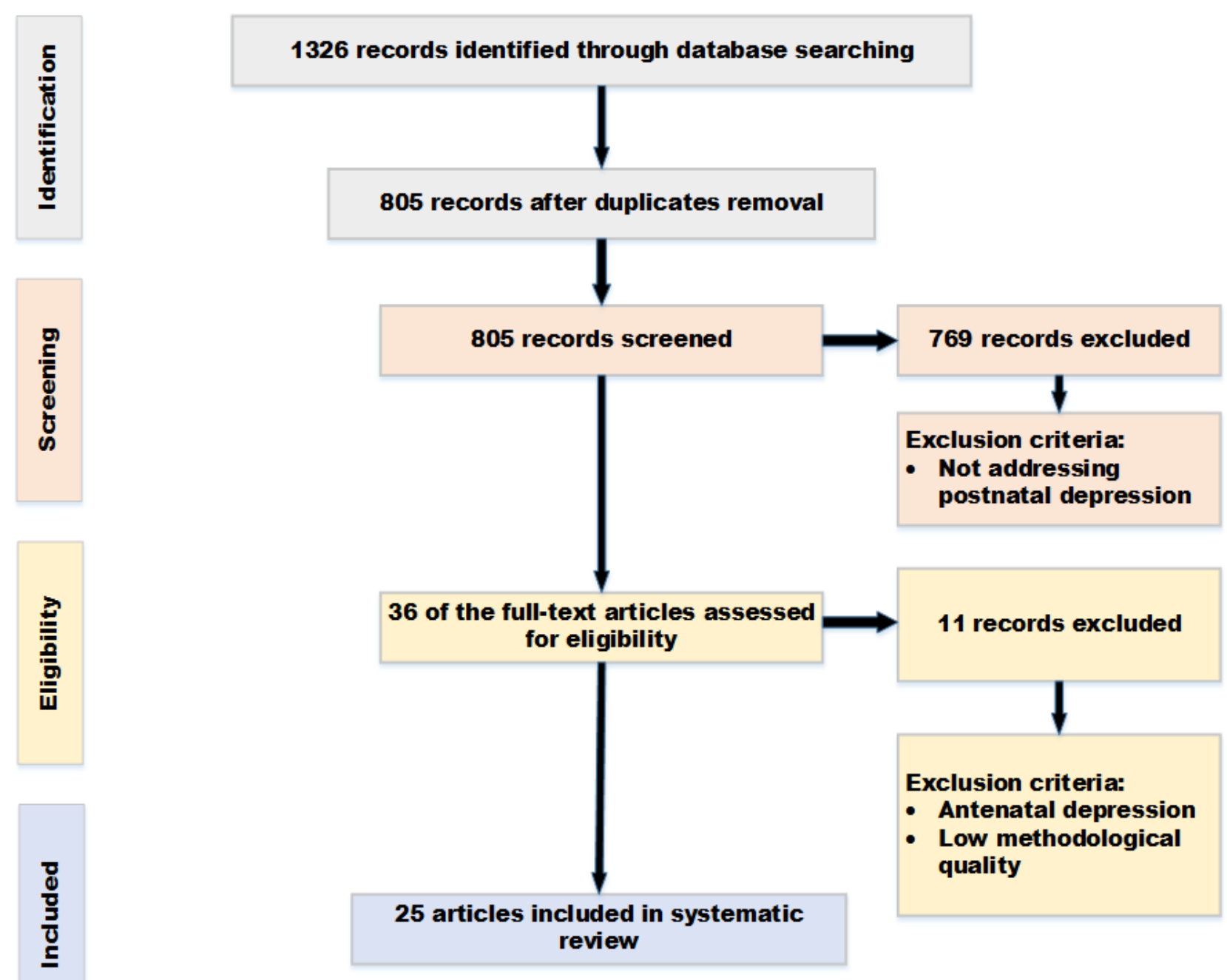

Figure 1: Search strategy flow diagram.

full-text article in English published from 2000 to 2016. Manually searching and reviewing references cited in retrieved articles supplemented the search (Figure 1).

\section{Study selection (Screening)}

Our study included measuring the methodological strength of each study based on a checklist developed by Mirza and Jenkins [43] and used by Sawyer, et al. [44]. Table 1 shows the methodological quality of the studies, with the following criteria, assessed: 1) Explicit aims, 2) Adequate and sample size justification, 3) Justification that sample is representative of population, 4) Clear inclusion and exclusion criteria, 5) Valid measurement of postnatal depression, 6) Response rate and drop out specified, 7) Adequate description of data, 8) Appropriate statistical analysis and 9) Delivery of intervention. The studies were then given a total score with the highest possible being nine $(1=$ Yes, $0=$ No). Table 2 displays the quality score for each study. Studies included in this review were of reasonable quality with over $80 \%$ of studies having a score of 7 or more. The types of intervention measured by studies included in this review were delivered by primary care practitioners [37]; GPs [37]; Health Visitors (HV) [45]; computer-based, telephone-based [46] and Internet-based interventions [19].

\section{Data extraction and synthesis}

For each article, the intervention used varied between studies. For this review, treatments, and interventions have been grouped into Pharmacological (PMI), which can include any antidepressants. Psychological Interventions (PHI) include Cognitive Behavioural Therapy; Computerized Cognitive Behavioural Therapy; Problem Solving Therapy and Interpersonal Therapy. Multicomponent interventions (MCP) includes peer support; exercise based; monitoring and assistance; educational programme and listening visit [47] and finally Usual/Routine Care (UC). The majority of the studies included in this review were Randomized Controlled Trials (RCT), while the others were a Cross-Sectional Survey (CSS). Five different measurement scale for depression were used, EPDS Edinburgh Postnatal Depression Scale (EPDS); Beck's Depression Inventory (BDI); Montgomery-Asberg Depression Rating Scale (MADRS); Hamilton Rating Scale for Depression (HRS-D) and Diagnostic and Statistical Manual of Mental Disorders, $4^{\text {th }}$ edition (DSMI-IV). Following Pampallona and colleagues [22] 


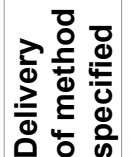

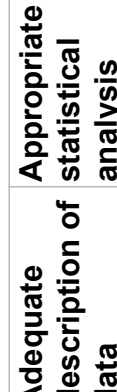

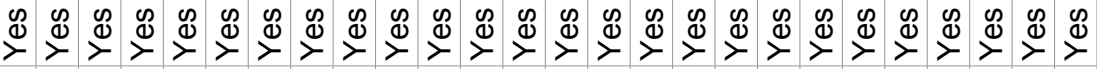

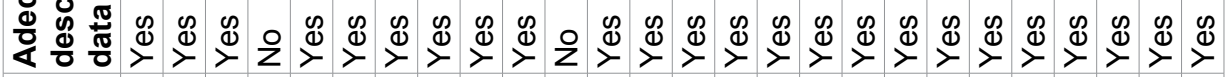

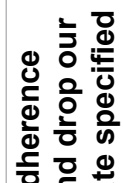

妾

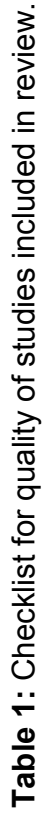

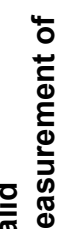

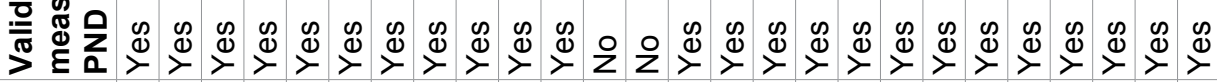

$\frac{0}{\frac{0}{0}} \frac{0}{0}$

造

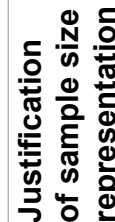

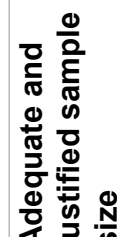

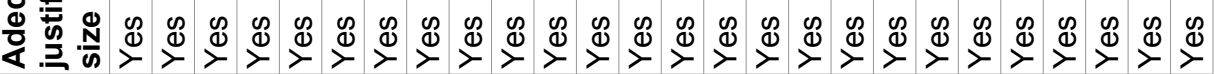

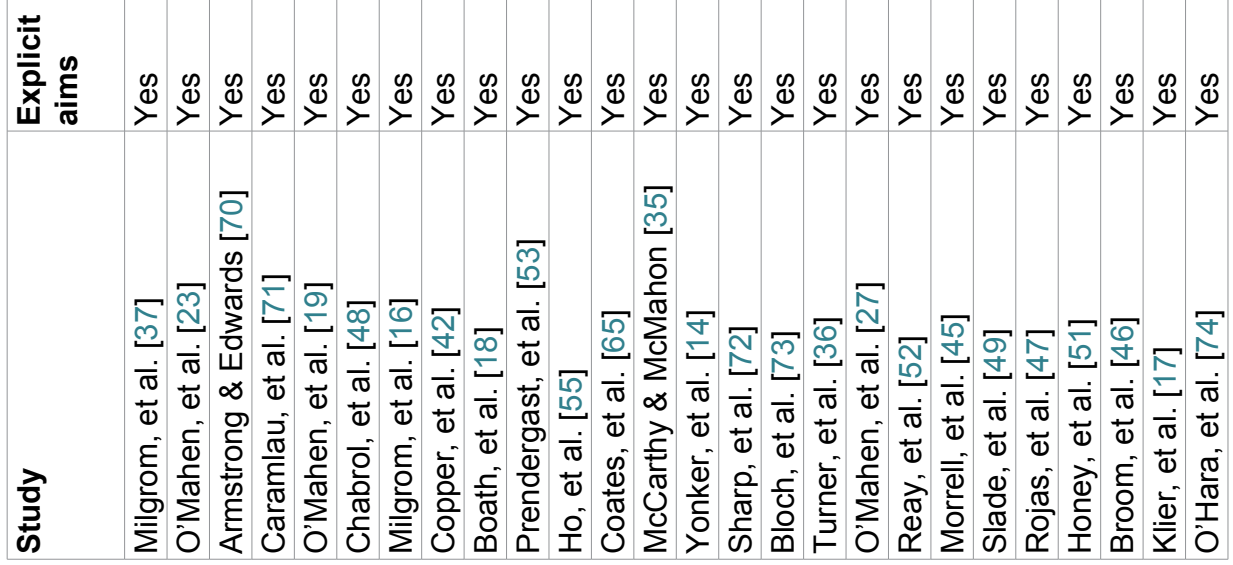


approach, the measures of adherence were grouped into four categories. a) Appointments kept (whether patients respected a pre-set schedule), b) Pills monitoring (any measures of adherence based on direct count of pills actually taken), c) Protocol deviation (termination of treatment before planned treatment period), d) Composite index (a scale comprising of any measures of intake of drug and other indicators such as patient satisfaction, reasons for stopping treatment, knowledge about drugs, satisfaction with treatment).

\section{Results}

\section{Study characteristics}

Twenty-five studies were included in the review with a total of 2438 participants. Sample size ranged from 15 to 595 (mean $=116, \mathrm{Std}=132.6$, mode $=17)$. The majority of the studies were conducted in the United Kingdom ( $n=11)$ followed by Australia $(n=4)$; United States $(n=$ $3)$; Israel $(n=1)$; Chile $(n=1)$; Austria $(n=1)$; Zimbabwe $(n=1)$; France $(n=1)$; Taiwan $(n=1)$ and New Zealand $(n$ $=1$ ). Four different approach of measuring depression was used by studies in this review: EPDS ( $n=15)$, DSMIV $(n=1), \operatorname{HDRS}(n=1)$ and BDI $(n=2)$. One study did not record the method used $(n=1)$, another study assessed depression through a clinical interview $(n=1)$ while the remaining studies assessed participants using multiple approaches $(n=6)$.

Ten studies were delivered at the Primary Care Centre (PCC) $(n=10)$ and seven at the Participant's Home $(\mathrm{PH})(\mathrm{n}=7)$. Four studies were conducted at the $\mathrm{PH}$ and PCC $(n=4)$, and four studies did not record the intervention location $(n=4)$. Eighteen studies delivered the intervention Face to Face (FF) $(n=18)$, one study via the Participant's Telephone (PT) $(n=1)$, while three other studies used a combination of both FF and the PT ( $n=$ 3). One study provided the intervention through PT and email $(n=1)$, another study online $(n=11)$ and lastly one study delivered the intervention by combining PT and online $(n=1)$.

For this paper, Health Practitioner (HP) includes therapist; psychiatrist; GP and clinicians. Ten of the studies delivered intervention with the help of HP $(n=10)$, and five studies used Health Visitor (HV) $(n=5)$. While three studies used a combination of HP and HV $(n=3)$ another study used $\mathrm{HV}$ and email ( $n=1)$. In two studies interventions was administered via text messages ( $n=2)$, two studies were Routine Care $(R C)(n=2)$, one study was facilitated by the Primary Care Nurse (PCN) $(n=1)$ and finally a combination of PCN and self-help $(n=1)$.

Two studies measured adherence by using pills monitoring ( $n=2$ ) while five studies utilised protocol deviation $(n=5)$. Appointments kept were used in two studies $(n=2)$, and five studies measured composite index $(n=5)$. One study used a combination of protocol deviation and composite index $(n=1)$, another study a com- bination of protocol deviation and pill monitoring ( $\mathrm{n}=$ 1). One study used a combination of protocol deviation, pill monitoring and appointment kept $(n=1)$. However, eight studies were not explicit on how they measured women's compliance to treatment $(n=8)$ but provided clues on the association between the delivery of PND treatment and adherence.

Eighteen studies were randomised control trials ( $\mathrm{n}$ $=18$ ), while eleven were Cross-Sectional Studies (CSS) ( $n=11$ ), and six studies used a combination of RCT and CSS $(n=6)$. Four studies used MCP $(n=4)$, two studies used PMI $(n=2)$, and four studies used PHI $(n=4)$. Some of these studies combined interventions, MCP and PMI $(\mathrm{n}=4)$; MCP and $\mathrm{PHI}(\mathrm{n}=6) ; \mathrm{MCP}, \mathrm{PHI}$ and $\mathrm{PMI}(\mathrm{n}=1)$; MCP and UC ( $n=1)$; PHI and UC $(n=2)$. However, one study did not record the type of intervention used for their research protocol. All participants were women with postnatal depression. Table 2 gives an overview of the study described characteristics.

\section{Discussion}

This review provides an overview of the current knowledge on the factors that determine the association between PND intervention delivery and its subsequent adherence. This report includes studies from several countries published over a 16-year period. Several indications suggest that PND intervention delivery is associated with its subsequent adherence.

\section{Factors that determine the association between in- tervention delivery and its subsequent adherence}

In this section, we highlight the factors that could determine the effect of intervention delivery approach on its subsequent adherence. The delivery of effective treatment is considered a priority and some treatments are delivered face to face at PH or PCC $[35,45,48-50]$. This method of delivery is effective and provides better psychological adjustments, but it can be costly and time intensive for providers and women, reducing the capacity of reach of these treatments $[5,11,23,32]$. This indicates that the delivery cost and times could potentially impact the delivery prescribed PND treatments and adherence. Two studies that delivered intervention with the help of HV suggested that good communication leads to a commitment to health provider's advice and prescribed therapy and could result in greater adherence $[49,50]$. Therefore, communication is crucial while delivering treatments.

Furthermore, studies that provided childcare during intervention process established an increased level of adherence [47,51,52]. Adequate provision for infant care during treatment could be an adherence factor. Also, three studies showed that adherence might be linked to the location in which the treatment is delivered $[48,52,53]$. This is seen to have a relationship with stress management, and this might be due to transportation 
difficulties faced by some women [25]. In regards to location, studies have documented the inability of women to attend regularly scheduled appointments [23]. Some studies demonstrated that delivery and adherence to intervention could be dependent on the duration of intervention process $[47,48,50,54]$. Two studies highlighted that women's engagement with the intervention might improve adherence to treatment $[37,55]$. Four studies that provided a convenient intervention time showed that a flexible delivery choice and arrangement is associated with increased adherence $[17,23,49,52]$.

\section{Does intervention delivery approach improve ad- herence to treatment?}

The review indicated that the intervention delivery approach could impact adherence to prescribed PND treatment $[26,35,45,47,50,52,56-60]$. Women with PND have found the presence of HV's valuable. This might be because HV's can empathise with women experiencing PND while delivering enhanced psychological care, with monitoring in place, upheld adherence and [37,45,61]. Moreover, women commented that having listening visits delivered by $\mathrm{HV}$ at home meant that they could talk in private with HV [50]. However, these studies have not taken into account the limited availability of human resources [50,62]. Time commitments are required from both health workers and women who can be filled with demanding responsibilities. It is likely that some women will not receive regular support due to the HV's absence [45]. Furthermore, practitioners providing visits need to have the time and skills to listen and provide tailored support [50].

An alternative to home-based HV delivered intervention is the provision of group treatment for PND. The group delivered intervention provides depressed women with the opportunity to share their experience of adjusting to motherhood or difficulties they go through with their peers and can help to reduce any feelings of solitude and loneliness $[17,52]$. Socially isolated postnatal women particularly benefit from the support and empathy provided by the group, which can break patterns of social isolation and increase their recognition of the detrimental effect, which can result from self-stigmatisation [17]. These studies give important clues on the direction of the association between delivery and adherence as they present evidence that suggests depressed women's engagement with treatment could act as an important factor and thus might improve adherence $[37,55,63,64]$.

\section{Does depressed women's engagement with inter- vention improve adherence to treatment?}

Women are more likely to adhere to the treatment if they can engage with treatment facilitator and are extremely positive about their relationships with their health visitor or the delivery approach $[37,55]$. This is consistent with the study that revealed that it was only after women got into a supportive treatment relationship and improved their communication that they felt able to disclose their distress to others [35]. However, questions need to be asked on how to engage women if additional treatment options are made available.

\section{Does intervention augmented by an adjunct support mechanism improve depressed women's adherence to treatment?}

This review has identified that interventions that are augmented by an adjunct support mechanism have the potential to improve adherence $[37,47]$. From the literature women receiving weekly phone calls, emails, and text message as additional support to treatment they received have found this helpful and reported high compliance rate $[2,28,42,46,50,65]$. Similarly, there was a record of increased adherence in a multicomponent intervention that was supported by regular phone calls from trained mental health workers. In this study, depressed women were always reminded about the need for taking medications as prescribed in treatment sessions [47]. However, lack of consultation time is highlighted as unsatisfactory and women would prefer better follow-up care from health professionals [18]. It is advised that adjunct treatment needs to remain available to women receiving visits both during and after the visit has ended [50]. This might be a solution to the disappearance of improvement recorded at 3-4.5 months at 6-12 month in a study on the short- and long-term effect of psychological treatment of PND as well as improve adherence [42,47].

\section{Does provision of infant care during intervention process improve adherence to treatment?}

Women are faced with the problems of managing their infant's needs while balancing other valued tasks [19]. There is evidence that adequate provision for infant care plays a vital role in the association between intervention delivery and adherence to the treatment plan [52]. Studies that provided facilities for infant care during treatment trials reported a higher level of compliance to treatment than those without $[47,51,52]$. However, additional empirical evidence is warranted, specifically prospective studies should take into account both the cost of delivery and childcare.

\section{Does a flexible intervention delivery improve ad- herence to treatment?}

A flexible delivery choice and arrangement have been associated with increased adherence [17,28,49,52]. Some women discounted the treatment because they did not have enough time, women who were working or studying completed fewer treatment modules while being on maternity leave or not working were related to completing more modules [28]. In a study that demonstrated increased adherence, of the 17 eligible participants, 15 were on maternity leave (commonly up to 2 
years in Australia), one was unemployed, and 1 was a housewife [17]. This showed that women the women on maternity were able to attend due to the reduced work commitments. Another study showed that treatment took place during school term to attract mothers with additional school-age children and minimise dropout [52]. Therefore a flexible delivery choice may contribute to quality delivery and increased adherence.

\section{Does depressed women's knowledge of the duration of intervention improve adherence to treatment?}

In a study, women commented that they knew they only had one hour to talk to their HV [55]. Depressed women's knowledge of the duration of intervention is a strong predictor of adherence [47,48,50,54,55]. However, there is limited empirical evidence to make a generalised conclusion.

\section{Limitations}

The studies included in this review used a range of measures and at different time points after childbirth and this makes it difficult to explain how treatment adherence and PND progresses over time. It is very difficult to distinguish those with first-time mental illness or previous history of mental health [66]. The majority of the studies used EPDS to identify depression. However, the cut-off points varied, leading to different estimation of the prevalence rate. The significant variance between study samples has implications for generalisability and interpretation of research findings. With regards to the measures of adherence, some studies used multiple measures and at different time points during the study. For example, in one study the administering of antidepressant was irregular based on participant's improvement, and compliance was assessed by a pill count conducted at each follow-up. One cannot exclude the possibility that the women manipulated themselves to appear compliant. Furthermore, some studies combined protocol deviation and appointment kept to assess adherence, this makes it difficult to conclude on the factors that motivate women, the impact of the adherence and if women were able to sustain it in the long-term. It is also important to note that there is a possibility that some of the women who completed the study might have been provided with additional support (such as support/treatments reminder from family) that can facilitate compliance with treatment.

While there are reasonable theoretical justifications for many of the intervention delivery approaches, their efficacy has not been established, and there is very little good evidence available on which to base policy or practice recommendations [67]. It is also not clear whether the delivery approach helped depressed mothers and her infant live their lives better or if the intervention had an effect on mothers experience immediately after treatment. There is also insufficient evidence to conclude on whether a depressed mother's preference of delivery approach can improve adherence. Also, research has not shown the procedures about the consistency of support especially in the case of absent healthcare workers.

This review highlights various omissions in published literature. For example, research on factors that determine women's engagement with treatment after childbirth is limited. This is a significant gap to be addressed, particularly as active engagement can enhance treatment adherence [26]. Likewise, there are few studies using adjunct support to treatment, which provides additional follow-up resulting in difficulty concluding its impact on PND treatment delivery and adherence. Therefore, further studies are required to establish the benefits of other support in improving adherence to PND treatments.

\section{Conclusion}

Non-adherence to treatment is a major problem faced by healthcare professionals and can increase the cost of service, both in financial terms and time investment [22]. A lack of adherence to treatment by women with PND has been shown to increase the length of time spent in treatment for psychological disorders, as well as to decrease the long-term effect of the treatment outcome $[42,54,68]$. However, non-adherence should not be entirely seen as the patient's problem as it represents a fundamental limitation in the delivery of healthcare [19]. This review has identified an association between intervention delivery and adherence such as provision for infant care; flexible intervention delivery; availability of adjunct support to treatment; delivery duration; delivery approach and women's engagement with intervention delivery. However, a comprehensive study of this subject is needed to adequately ascertain which factors motivate women and identify obstacles that contribute to non-adherence. Furthermore, we advise research in providing adjunct support to PND treatments. This is because offering additional support may circumvent many of the barriers to treatment delivery, adherence and motivate women to self-manage their wellbeing. This could also give users access to additional wellbeing support.

In general, given the association between intervention delivery and adherence, it is increasingly seen as important to have in place adjunct intervention delivery that will help women comply with treatment and help sustain treatment effect over the long-term [42,47]. The United Kingdom National Health Service (NHS) suggests that better use of data and technology has the power to improve health, transforming the quality and reducing the cost of health and social care services. It can give patients and citizens more control over their health and wellbeing, empower practitioners, reduce the administrative burden for care professionals, and support the development of new medicines and treatments [69]. Further studies will be required to establish acceptable 
intervention delivery methods and adjunct support for women with PND.

This research did not receive any specific grant from funding agencies in the public, commercial, or not-forprofit sectors.

\section{Role of Funding Source}

No funding was provided for this study.

\section{Conflict of Interest}

This work has no conflict of interest.

\section{Acknowledgement}

There are no acknowledgements for this research.

\section{References}

1. Lewis G (2004) Why Mothers Die 2000-2002-The sixth report of confidential enquiries into maternal deaths in The Kingdom CEMACH London.

2. Cuijpers P, Brannmark JG, van Straten A (2008) Psychological treatment of postnatal depression: A meta-analysis. J Clin Psychol 64: 103-118.

3. Hewitt C, Gilbody S, Brealey S, Paulden M, Palmer S, et al. (2009) Methods to identify postnatal depression in primary care: An integrated evidence synthesis and value of information analysis. Health Technol Assess 13: 1-145.

4. Gibson J, McKenzie-McHarg K, Shakespeare J, Price J, Gray R (2009) A systematic review of studies validating the Edinburgh PND Scale in antepartum and postnatal women. Acta Psychiatr Scand 119: 350-364.

5. Stevenson MD, Scope A, Sutcliffe PA, Booth A, Slade P, et al. (2010) Group cognitive behavioural therapy for postnatal depression: A systematic review of clinical effectiveness, cost- effectiveness and value of information analyses. Health Technol Assess 14: 1-107.

6. Evans M, Donelle L, Hume-Loveland L (2011) Social support and online postnatal depression discussion groups: A content analysis. Patient Educ Couns 87: 405-410.

7. Morrell JC (2006) Review of interventions to prevent or treat postnatal depression. Clinical Effectiveness in Nursing 9: e135-e161.

8. NICE (2015) Postnatal Care. NICE Clinical Guideline 37: 1-67.

9. Graham C, Franses A, Kenwright M, Marks I (2000) Psychotherapy by computer: A postal survey of responders to a teletext article. Psychiatric Bulletin 24: 331-332.

10. Hoffbrand S, Howard L, Crawley H (2001) Antidepressant drug treatment for postnatal depression. Cochrane Database Syst Rev.

11. Kaltenthaler E, Parry G, Beverley C, Ferriter M (2008) Computerised cognitive-behavioural therapy for depression: Systematic review. Br J Psychiatry 193: 181-184.

12. Proudfoot J, Ryen C, Everitt B, Sharpio D, Goldberg D, et al (2004) Clinical efficacy of computerized cognitive-behavioural therapy for anxiety and depression in primary care: Randomised controlled trial. Br J Psychiatry 185: 46-54.

13. Misri S, Reebye P, Corral M, Mills L (2004) The use of paroxetine and cognitive-behavioral therapy in postnatal depression and anxiety: A randomized controlled trial. J Clin Psychiatry 65: 1236-1241.
14. Yonkers KA, Lin H, Howell HB, Heath AC, Cohen LS (2008) Pharmacological treatment of postnatal women with new onset major depressive disorder: A randomized controlled trial with paroxetine. J Clin Psychiatry 69: 659-665.

15. Molyneaux E, Howard LM, McGeown HR, Karia AM, Trevillion K (2014) Antidepressant treatment for postnatal depression. Cochrane Database Syst Rev.

16. Milgrom J, Negri LM, Gemmill AW, McNeil M, Martin PR (2005) A randomized controlled trial of psychological interventions for postnatal depression. Br J Clin Psychol 44: 529-542.

17. Klier CM, Muzik M, Rosenblum KL, Lenz G (2001) Interpersonal psychotherapy adapted for the group setting in the treatment of postnatal depression. J Psychother Pract Res 10: $124-131$

18. Boath E, Bradley E, Anthony P (2004) Users' views of two alternative approaches to the treatment of postnatal depression. Journal of Reproductive and Infant Psychology 22: 13-24.

19. O'Mahen HA, Richards DA, Woodford J, Wilkinson E, McGinley J, et al. (2013) Netmum, a phase II randomized controlled trial of guide Internet behavioural activation treatment for postnatal depression. Psychol Med 1-15.

20. Omisade O, Good A, Fitch T, Briggs J (2017) An analysis of factors affecting postnatal depression intervention adherence. International Journal of Public Health Management and Ethics (IJPHME) 2: 1-18.

21. Bruer JT (1982) Methodological rigor and citation frequency inpatient compliance literature. Am J Public Health 72: 1119-1123.

22. Pampallona S, Bollini P, Tibaldi G, Kupelnick B, Munizza $C$ (2002) Patient adherence in the treatment of depression. $\mathrm{Br}$ J Psychiatry 180: 104-109.

23. O'Mahen HA, Grieve H, Jones J, McGinley J, Woodford $J$, et al. (2015) Women's experience of factors affecting treatment engagement and adherence in Internet delivered Behavioral Activation for Postnatal depression. Internet Intervention 2: 84-90.

24. O'Mahen HA, Flynn HA (2008) Preferences and perceived barriers to treatment for depression during the perinatal period. J Womens Health (Larchmt) 17: 1301-1309.

25. Goodman JH (2009) Women's attitudes, preferences, and perceived barriers to treatment for perinatal depression. Birth 36: 60-69.

26. de Graaf LE, Gerhards SAH, Arntz A, Riper H, Metsemakers JF, et al. (2009) Clinical effectiveness of online computerised cognitive-behavioural therapy without support for depression in primary care: Randomised trial. $\mathrm{Br} \mathrm{J}$ Psychiatry 195: 73-80.

27. O'Mahen H, Fedock G, Henshaw E, Himle JA, Forman J, et al. (2011) Modifying CBT for perinatal depression: What do women want? Cognitive and Behavioral Practice.

28. O'Mahen HA, Richards SA, Woodford J, Wilkinson E, McGinley J, et al. (2014) Netmums: A phase II randomized controlled trial of guided Internet behavioural activation treatment for postnatal depression. Psychol Med 44: 1675-1689.

29. Dennis CL, Chung-Lee L (2006) Postnatal depression help-seeking barriers and maternal treatment preferences: A qualitative systematic review. Birth 33: 323-331.

30. Christensen H, Griffiths K, Farrer L (2009) Adherence in internet intervention for anxiety and depression: Systematic review. J Med Internet Res 1-16. 
31. Knudson-Martin C, Silverstein R (2009) Suffering in silence: A qualitative meta-data- analysis of postnatal depression. $J$ Marital Fam Ther 35: 145-158.

32. Gerhards SA, de Graaf LE, Jacobs LE, Severens LJ, Huibers MJ, et al. (2010) Economic evaluation of onilne computerised cognitive-behavioural therapy without support for depression in primary care: Randomised trial. $\mathrm{Br} \mathrm{J}$ Psychiatry 196: 310-318.

33. Merry SN, Stasiak K, Shepheard M, Frampton C, Fleming T, et al. (2012) The effectiveness of SPARX, a computerised self-help intervention for adolescents seeking help for depression: Randomised controlled non-inferiority trial. BMJ 344: 1-16.

34. Glover V, Onozawa K, Hodgkinson A (2002) Benefits of infant massage for mothers with postnatal depression. Semin Neonatol 7: 495-500.

35. McCarthy M, McMahon C (2008) Acceptance and experience of treatment for PND in a community mental health setting. Health Care Women Int 29: 618-637.

36. Turner KM, Sharp D, Folkes L, Chew-Graham C (2008) Women's views and experiences of antidepressants as a treatment for postnatal depression: A qualitative study. Fam Pract 25: 450-455.

37. Milgrom J, Holt CJ, Gemmill AW, Ericksen J, Leigh B, et al. (2011) Treating postnatal depressive symptoms in primary care: A randomised controlled trial of GP management, with and without adjunctive counseling. BMC Psychiatry 11: 95.

38. Dennis CL (2014) The process of developing and implementing a telephone-based peer support program for postnatal depression: Evidence from two randomised controlled trial. Trial 15: 131.

39. Gonzalez J, Williams JW Jr, Noël PH, Lee S (2005) Adherence to mental health treatment in a primary care clinic. $J$ Am Board Fam Pract 18: 87-96.

40. Kaplan WA (2006) Can the ubiquitous power of mobile phones be used to improve health outcomes in developing countries. Global Health 2: 9.

41. Dennis CL, Ravitz $P$, Grigoriadis $S$, Jovellanos $M$, Hodnett $E$, et al. (2012) The effect of telephone-based interpersonal psychotherapy for the treatment of postnatal depression: Study protocol for a randomized controlled trial. Trials 13: 38.

42. Cooper PJ, Murray L, Wilson A, Romaniuk H (2003) Controlled trial of the short- and long-term effect of psychological treatment of post-partum depression I. Impact on maternal mood. Br J Psychiatry 182: 412-419.

43. Mirza I, Jenkins R (2004) Risks factors, prevalence, and treatment of anxiety and depressive disorders in Pakistan: Systematic review. BMJ 328: 794.

44. Sawyer A, Ayers S, Smith H (2010) Pre- and postnatal psychological wellbeing in Africa: A systematic review. J Affect Disord 123: 17-29.

45. Morrell CJ, Slade P, Warner R, Paley G, Dixon S, et al. (2009) Clinical effectiveness of health visitor training in psychologically informed approaches for depression in postnatal women: Pragmatic cluster randomised trial in primary care. BMJ 338: 3045.

46. Broom MA, Ladley AS, Rhyne EA, Halloran DR (2015) Feasibility and perception of using text messages as an adjunct therapy for low-income, minority mothers with postnatal depression. JMIR Ment Health 2: e4.

47. Rojas G, Fritsch R, Solis J, Jadresic E, Castillo C, et al.
(2007) Treatment of postnatal depression in low-income mothers in primary-care clinics in Santiago, Chile: A randomized controlled trial. Lancet 370: 1629-1637.

48. Chabrol H, Teissedre F, Saint-Jean M, Teisseyre N, Rogé B, et al. (2002) Prevention and treatment of post-partum depression: A controlled randomized study on women at risk. Psychol Med 32: 1039-1047.

49. Slade P, Morrell CJ, Rigby A, Ricci K, Spittlehouse J, et al. (2010) Postnatal women's experiences of management of depressive symptoms: A qualitative study. $\mathrm{Br} \mathrm{J}$ Gen Pract 60: e440-e448.

50. Turner KM, Chew-Graham C, Folkes L, Sharp D (2010) Women's experiences of health visitors delivered listening visits as a treatment for postnatal depression: A qualitative study. Patient Educ Couns 78: 234-239.

51. Honey KL, Bennett P, Morgan M (2002) A brief psycho-educational group intervention for postnatal depression. $\mathrm{Br} \mathrm{J}$ Clin Psychol 41: 405-409.

52. Reay R, Fisher Y, Robertson M, Adams E, Owen C, et al. (2006) Group interpersonal psychotherapy for postnatal depression: A pilot study. Arch Womens Ment Health 9: 31-39.

53. Prendergast J, Austin M (2001) Early childhood nurse-delivered cognitive behavioural counselling for post-natal depression. Australasian Psychiatry 9: 255-259.

54. Rigbi A, Shalev-Mevorach L, Taller A, Taller Y, Lerer B (2003) Relationship of clinical and demographic characteristics of schizophrenia patients to rehabilitation status. Isr J Psychiatry Relat Sci 40: 258-267.

55. Ho SM, Heh SS, Jevitt CM, Huang LH, Fu YY, et al. (2009) Effectiveness of discharged education in reducing the severity of postpartum depression: A randomized controlled evaluation study. Patient Educ Couns 77: 68-71.

56. Taggart AV, Short SD, Barclay L (2000) 'She has made me feel human again': An evaluation of a volunteer homebased visiting project for mothers. Health Soc Care Community 8: 1-8.

57. Dennis CL (2003) The effect of peer support on postnatal depression: A pilot randomized controlled trial. Can J Psychiatry 48: 115-124.

58. Scrandis DA (2005) Normalizing postnatal depressive symptoms with social support. Journal of the American Psychiatric Nurses Association 11: 223-230.

59. Milgrom J, Ericksen J, McCarthy R, Gemmill AW (2006) Stressful impact of depression on early mother-infant relations. Stress \& Health 22: 229-238.

60. Shaw E, Levitt C, Wong S, Kaczorowski J, McMaster University Postpartum Research Group (2006) Systematic review of the literature on postnatal care: Effectiveness of postnatal support to improve maternal parenting, mental health, quality of life, and physical health. Birth 33: 210-220.

61. Murray L, Cooper P, Fearon P (2014) Parenting difficulties and postnatal depression: Implication for primary healthcare assessment and intervention. Community Pract 87 : 34-38.

62. Sikander S, Lazarus A, Bangash O, Fuhr DC, Weobong $B$, et al. (2015) The effectiveness and cost-effectiveness of the peer-delivered thinking healthy programme for peri-natal depression in Pakistan and India: The SHARE study protocol for randomised controlled trail. Trials 16: 534.

63. Scholle SH, Kelleher K (2003) Preferences for depression advice among low-income women. Matern Child Health $\mathrm{J}$ 7: 95-102. 
64. Lin P, Campbell DG, Chaney EF, Liu CF, Heagerty P, et al. (2005) The influence of patient preference on depression treatment in primary care. Ann Behav Med 30: 164-173.

65. Coates R, Ayers S, de Visser R (2014) Women's experiences of postnatal distress: A qualitative study. BMC Pregnancy Childbirth 14: 359.

66. Kessler RC (2003) Epidemiology of women and depression. J Affect Disord 74: 5-13.

67. Boath E, Henshaw C (2001) The treatment of postnatal depression: A comprehensive literature review. Journal of Reproductive and Infant Psychology 19: 215-248.

68. Dunn G (2002) The challenge of patient choice and nonadherence to treatment in randomized controlled trials of counselling or psychotherapy. Understanding Statistics 1: 19-29.

69. Personalised Health and Care 2020 (2014) Using data and technology to transform outcome for patients and citizens: A framework for action. NHS.

70. Armstrong K, Edwards $\mathrm{H}$ (2004) The effectiveness of a pram-walking exercise programme in reducing depressive symptomatology for postnatal women. Int J Nurs Pract 10: 177-194.

71. Caramlau I, Barlow J, Sembi S, McKenzie-McHarg K, McCabe C (2011) Mums 4 Mums: Structured telephone peer-support for women experiencing PND. Pilot and exploratory RCT of its clinical and cost effectiveness. Trials 12: 88.

72. Sharp DJ, Chew-Graham C, Tylee A, Lewis G, Howard L, et al. (2010) A pragmatic randomised controlled trial to compare antidepressants with a community-based psychosocial intervention for the treatment of women with postnatal depression: The RESPOND trial. Health Technol Assess 14: 1-53.

73. Bloch M, Meiboom H, Lorberblatt M, Bluvstein I, Aharonov I, et al. (2012) The effect of sertraline add-on to brief dynamic psychotherapy for the treatment of postpartum depression: A randomized, double-blind, placebo-controlled study. J Clin Psychiatry 73: 235-241.

74. O'Hara MW, Stuart S, Gorman LL, Wenzel A (2000) Efficacy of interpersonal psychotherapy for postpartum depression. Arch Gen Psychiatry 57: 1039-1045. 